\title{
Vertex Polynomial of Middle, Line and Total Graphs of some standard Graphs
}

\author{
Mallikarjun Basanna Kattimani, Sridhara K.R.
}

\begin{abstract}
In this article, we have obtained vertex polynomial of Middle, Line and Total graphs of some standard graphs namely complete graph, star, path, cycle and wheel graph.

Keywords: Vertex polynomial, Middle graph, Line Graph, Total Graph
\end{abstract}

\section{I.INTRODUCTION}

In this paper, we have considered only finite graphs which are non-trivial, undirected, having no self- loops and having no parallel edges. Two edges of a graph are adjacent if they have a common vertex. If a vertex $v$ is an end vertex of an edge $e$, then edge $e$ is incident on vertex $v$. Vertices and edges are called elements of a graph and are neighbors if they are either incident or adjacent. $K_{1, n}$ represents a star. A wheel graph $W_{n}$ and is defined as $W_{n}=K_{1}+C_{n-1}$. A graph $G$ is $k$ - regular if each vertex has degree $k$. If $x=u v$ is an edge of a graph $G$, and $w$ is not a vertex of $G$, then $x$ is said to subdivided if it is replaced by the edges $u w$ and $v w$. For terminologies and notations, we refer [1]. The vertex polynomial of the graph $G$ [2] is defined as $V(G ; x)=$ $\sum_{k=0}^{\triangle(G)} v_{k} x^{k}$, where $\triangle(G)=\max \{d(v): v \in V(G)\}$ and $v_{k}$ is the number of vertices of degree $k$. The roots of a vertex polynomial are called vertex polynomial roots.

\section{II.MIDDLE GRAPH, LINE GRAPH, TOTAL GRAPH AND VERTEX POLYNOMIAL WITH AN EXAMPLE.}

In this part, we list the definitions of Middle graph, Line graph and Total graph of a graph with an example. Also we show their vertex polynomials.

Let $G$ be a graph with $|V|=m$ and $|E|=n$. Its line graph $L(G)$ contains vertices which are edges of $G$ and edges in $L(G)$ are drawn between vertices if they have common vertex in $G$. Middle graph $M(G)$ has vertex set containing all vertices and all edges of $G$ and edges of $M(G)$ between new vertices are drawn only when the edges corresponding in $G$ are adjacent. Total graph $T(G)$ has vertex set containing all vertices of $G$ and all edges of $G$ and two vertices of total graph are adjacent if they happen to be neighbors in $G$. The following figures show a graph, its line graph, middle graph and total graph with their vertex polynomials.

Revised Manuscript Received on April 03, 2020.

* Correspondence Author

Mallikarjun Basanna Kattimani, Department of Mathematics, The

Oxford College of Engineering, Bangalore, India, mathshodoxford@gmail.com

Sridhara K.R.*, Department of Mathematics, A.P.S. College of Engineering, Bangalore, India, sridharwi@gmail.com

(c) The Authors. Published by Blue Eyes Intelligence Engineering and Sciences Publication (BEIESP). This is an open access article under the CC BY-NC-ND license (http://creativecommons.org/licenses/by-nc-nd/4.0/)

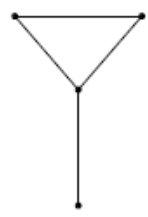

Figure 1- Graph $\boldsymbol{G}$.

In this graph, we find 1,2 and 1 vertices of degrees 1,2 and 3 respectively. Thus $V(G ; x)=x+2 x^{2}+x^{3}$. Now we consider its line graph as in Figure 2.

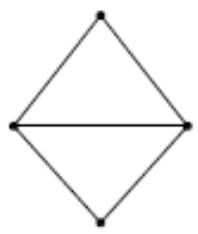

Figure 2- $\boldsymbol{L}(\boldsymbol{G})$

We note that $L(G)$ has four vertices of which two are of degree 2 and remaining two are of degree 3 . Thus $V(L(G) ; x)=2 x^{2}+2 x^{3}$

Now, we consider middle graph $M(G)$ as shown in Figure 3 .

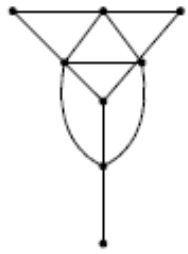

Figure 3-M(G)

The middle graph has one vertex of degree 1 , two vertices of degree 2 , one vertex of degree 3 , two vertices of degree 4 and two vertices of degree 5 . Thus $V(M(G) ; x)=x+2 x^{2}+$ $x^{3}+2 x^{4}+2 x^{5}$. Now we consider total graph $T(G)$ as shown in Figure 4.

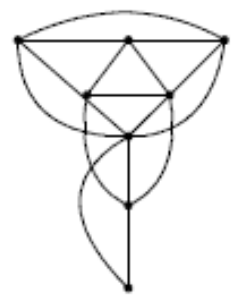

Figure 4-T(G)

Published By: Blue Eyes Intelligence Engineering \& Sciences Publication

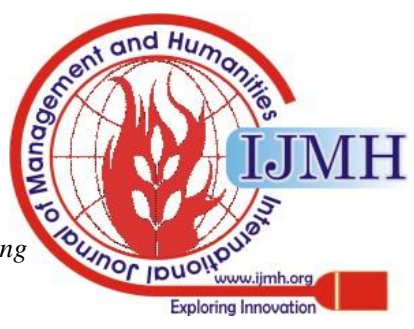




\section{Vertex Polynomial of Middle, Line and Total Graphs of some standard Graphs}

$T(G)$, the total graph has1, 4, 2 and 1 vertices of degrees 2, 4, 5 and 6 respectively.

Thus $V(T(G) ; x)=x^{2}+4 x^{4}+2 x^{5}+x^{6}$

\section{III.MAIN RESULTS}

Theorem 3.1: If $M\left(K_{n}\right)$ is middle graph of $K_{n}$, then

$$
V\left(M\left(K_{n}\right) ; x\right)=n x^{n-1}+\frac{n(n-1)}{2} x^{2 n-2}
$$

Proof: We note that $K_{n}$ has $n$ vertices, whereas its middle graph $M\left(K_{n}\right)$ has $\frac{n^{2}+n}{2}$ vertices by definition of middle graph. We can partition vertex set of $M\left(K_{n}\right)$ into two disjoint subsets $V_{1}$ and $V_{2}$ as follows:

$V_{1}$ : Vertex set with $\left|V_{1}\right|=n$, degree being $(n-1)$ for each vertex and

$V_{2}$ : Vertex set with $\left|V_{2}\right|=\frac{n^{2}-n}{2}$, degree being $(2 n-2)$ for each vertex.

Hence $V\left(M\left(K_{n}\right) ; x\right)=n x^{n-1}+\frac{n(n-1)}{2} x^{2 n-2}$

Theorem 3.2: If $M\left(K_{1, n}\right)$ is middle graph of $K_{1, n}$, then $V\left(M\left(K_{1, n}\right) ; x\right)=n x^{n+1}+x^{n}+n x$ for $n \geq 2$

Proof: We note that $K_{1, n}$ has $(n+1)$ vertices, whereas its middle graph $M\left(K_{1, n}\right)$ has $(2 n+1)$ vertices by definition of middle graph. We can partition the vertex set of $M\left(K_{1, n}\right)$ into disjoint subsets $V_{1} V_{2}$ and $V_{3}$ as follows:

$V_{1}$ : Vertex set containing 1 vertex of degree $n$,

$V_{2}$ : Vertex set with $\left|V_{2}\right|=n$, each vertex being pendant and

$V_{3}$ : Vertex set $\left|V_{3}\right|=n$,degree being $(n+1)$ for each vertex.

Thus $V\left(M\left(K_{1, n}\right) ; x\right)=n x^{n+1}+x^{n}+n x$

Theorem 3.3: If $M\left(P_{n}\right)$ is the middle graph of $P_{n}$, then $V\left(M\left(P_{n}\right) ; x\right)=2 x+(n-2) x^{2}+(n-1) x^{3}, n \geq 3$

Proof: We note, by definition of middle graph that, $M\left(P_{n}\right)$ contains $(2 n-1)$ vertices. We can partition the vertex set of $M\left(P_{n}\right)$ into three disjoint subsets $V_{1} V_{2}$ and $V_{3}$ as follows:

$V_{1}$ : Vertex set containing 2 vertices, each of degree 1 ,

$V_{2}$ : Vertex set with $\left|V_{2}\right|=n-2$, degree being 2 for each vertex and

$V_{3}$ : Vertex set with $\left|V_{3}\right|=n-1$, degree being 3 for each vertex.

Thus $V\left(M\left(P_{n}\right) ; x\right)=2 x+(n-2) x^{2}+(n-1) x^{3}$

Theorem 3.4: If $M\left(C_{n}\right)$ is the middle graph of $C_{n}$, then $V\left(M\left(C_{n}\right) ; x\right)=n x^{2}+n x^{4}$
Proof: We note, by definition of middle graph that, $M\left(C_{n}\right)$ contains $2 n$ vertices. We can partition the vertex set of $M\left(C_{n}\right)$ into $V_{1}$ and $V_{2}$ as follows:

$V_{1}$ : Vertex set containing $n$ vertices of $C_{n}$, each of degree 2 and

$V_{2}$ : Vertex set containing $n$ new vertices obtained by definition of middle graph, each of degree 4 .

Thus $V\left(M\left(C_{n}\right) ; x\right)=n x^{2}+n x^{4}$

Corollary 3.5: The vertex polynomial roots of $M\left(C_{n}\right)$ are 0 , 0 and $\pm i$

Proof: In view of Theorem 3.4, vertex polynomial roots of $M\left(C_{n}\right)$ can be obtained by taking

$$
n x^{2}+n x^{4}=0 \quad \Rightarrow \quad x=0,0 \text { and } \pm i
$$

Theorem 3.6: If $M\left(W_{n}\right)$ is the middle graph of $W_{n}$, then $V\left(M\left(W_{n}\right) ; x\right)=(n-1) x^{n+2}+x^{n-1}+(n-1) x^{6}+$ $(n-1) x^{3}$, for $n \geq 5$

Proof: We note, by definition of middle graph that, $M\left(W_{n}\right)$ contains $(3 n-2)$ vertices. We can partition vertex set of $M\left(W_{n}\right)$ into four disjoint subsets $V_{1}, V_{2}, V_{3}$ and $V_{4}$ as follows:

$V_{1}$ : Vertex set containing a single vertex of degree $(n-1)$,

$V_{2}$ : Vertex set with $\left|V_{2}\right|=n-1$, degree being 3 for each vertex,

$V_{3}$ : Vertex set $\left|V_{3}\right|=n-1$, degree being 6 for each vertex, and

$V_{4}$ : Vertex set $\left|V_{4}\right|=n-1$, degree being $n+2$ for each vertex.

Thus $V\left(M\left(W_{n}\right) ; x\right)=(n-1) x^{n+2}+x^{n-1}+(n-1) x^{6}+$ $(n-1) x^{3}$

Theorem 3.7: If $L\left(K_{n}\right)$ is the line graph of $K_{n}$, then

$$
\left.V\left(L\left(K_{n}\right) ; x\right)\right)=\frac{n(n-1)}{2} x^{n}
$$

Proof: Using definition of line graph, we easily understand that $L\left(K_{n}\right)$ is a $n$ - regular graph with $\frac{n^{2}-n}{2}$ vertices and every vertex will be of degree $n$.

Thus $V\left(L\left(K_{n} ; x\right)\right)=\frac{n(n-1)}{2} x^{n}$.

Theorem 3. 8: If $L\left(K_{1, n}\right)$ is the line graph of $K_{1, n}$, then $\left.V\left(L\left(K_{1, n}\right) ; x\right)\right)=n x^{n-1}$

Proof: By definition of line graph, of a graph, we easily understand that $L\left(K_{1, n}\right)$ is a complete graph $K_{n}$ and therefore every vertex will be of degree $(n-1)$. and $\mathrm{Hu}$ 
Thus $V\left(L\left(K_{1, n} ; x\right)\right)=n x^{n-1}$.

Theorem 3.9: If $L\left(P_{n}\right)$ is the line graph of $P_{n}$, then $\left.V\left(L\left(P_{n}\right) ; x\right)\right)=2 x+(n-3) x^{2}, n \geq 3$

Proof: By definition of line graph, we easily understand that $L\left(P_{n}\right)$ is a path graph $P_{n-1}$.

Thus $\left.V\left(L\left(P_{n}\right) ; x\right)\right)=V\left(P_{n-1} ; x\right)$.

It is easy to note that path graph $P_{n-1}$ will have 2 pendant vertices and remaining $(n-3)$ internal vertices will be of degree 2.

Thus $\left.V\left(L\left(P_{n}\right) ; x\right)\right)=2 x+(n-3) x^{2}$. $\left.V\left(L\left(C_{n}\right) ; x\right)\right)=n x^{2}$.

Proof: By definition of line graph , we easily understand that $L\left(C_{n}\right)=C_{n}$ itself. As degree of every vertex of $C_{n}$ is 2 and as it has $n$ vertices, we find $\left.V\left(L\left(C_{n}\right) ; x\right)\right)=n x^{2}$.

Corollary 3.11: The vertex polynomial root of $L\left(C_{n}\right)$ is 0 with multiplicity 2.

Proof: In view of Theorem 3.10, vertex polynomial roots of $L\left(C_{n}\right)$ can be obtained by taking

$$
n x^{2}=0 \quad \Rightarrow \quad x=0,0 \text { as } n \neq 0
$$

Theorem 3.12: If $L\left(W_{n}\right)$ is the line graph of $W_{n}$, then

$$
V\left(L\left(W_{n} ; x\right)\right)=(n-1) x^{4}+(n-1) x^{n}, \quad n \geq 4
$$

Proof: We know, a wheel graph $W_{n}$ will have $n$ vertices and $(2 n-2)$ edges. The vertex set of $L\left(W_{n}\right)$ will have $(2 n-2)$ vertices and we can partition the vertex set into two disjoint subsets $V_{1}$ and $V_{2}$ as follows:

$V_{1}$ : Vertex set with $\left|V_{1}\right|=n-1$, degree being 4 for each vertex

$V_{2}$ : Vertex set with $\left|V_{2}\right|=n-1$, degree being $n$ for each vertex

Thus $V\left(L\left(W_{n} ; x\right)\right)=(n-1) x^{4}+(n-1) x^{n}$

Theorem 3.13: If $T\left(K_{n}\right)$ is the total graph of $K_{n}$, then

$$
V\left(T\left(K_{n}\right) ; x\right)=\frac{n(n+1)}{2} x^{2 n-2}
$$

Proof: We note that $K_{n}$ will have $n$ vertices and $\frac{n(n-1)}{2}$ edges. By definition of total graph of a graph, we note that, total graph $T\left(K_{n}\right)$ will have $n+\frac{n(n-1)}{2}=\frac{n(n+1)}{2}$ vertices and each vertex will be of degree $(2 n-2)$.

Thus

$$
V\left(T\left(K_{n}\right) ; x\right)=\frac{n(n+1)}{2} x^{2 n-2}
$$

Theorem 3.14: If $T\left(K_{1, n}\right)$ is the total graph of $K_{1, n}$, then

$$
V\left(T\left(K_{1, n} ; x\right)=n x^{2}+n x^{n+1}+x^{2 n}\right.
$$

Theorem 3.10: If $L\left(C_{n}\right)$ is the line graph of $C_{n}$, then is

Proof: We know a star graph $K_{1, n}$ will have $n+1$ vertices and $n$ edges. We note that total graph $T\left(K_{1, n}\right)$ of star graph $K_{1, n}$ will have $(2 n+1)$ by the way in which total graph is defined and we can partition this the vertex set into 3 disjoint subsets $V_{1}, V_{2}$ and $V_{3}$ as follows:

$V_{1}$ : Vertex set containing a single vertex of degree $2 n$,

$V_{2}$ : Vertex set with $\left|V_{2}\right|=n-1$, degree being 2 for each vertex and

$V_{3}$ : Vertex set $\left|V_{3}\right|=n$, degree being 2 for each vertex. Thus $V\left(T\left(K_{1, n} ; x\right)=n x^{2}+n x^{n+1}+x^{2 n}\right.$

Theorem 3.15: If $T\left(P_{n}\right)$ is the total graph of $T\left(P_{n}\right)$, then $V\left(T\left(P_{n}\right) ; x\right)=2 x^{2}+2 x^{3}+(2 n-5) x^{4}, n \geq 3$

Proof: We know, a path graph $P_{n}$ will have $n$ vertices and $(n-1)$ edges. By definition of total graph, we note h $T\left(P_{n}\right)$ will have $(2 n-1)$ vertices and we can partition this vertex set into 3 disjoint subsets $V_{1}, V_{2}$ and $V_{3}$ as follows:

$V_{1}$ : Vertex set containing 2 vertices, each of degree 2 ,

$V_{2}$ : Vertex set containing 2 vertices, each of degree 3 and

$V_{3}$ : Vertex set containing $(2 n-5)$ vertices, each of degree 4 .

Thus $V\left(T\left(P_{n}\right) ; x\right)=2 x^{2}+2 x^{3}+(2 n-5) x^{4}$

Theorem 3.16: If $T\left(C_{n}\right)$ is the total graph of $C_{n}$, then $V\left(T\left(C_{n}\right) ; x\right)=2 n x^{4}$

Proof: We know, a cycle graph $C_{n}$ has $n$ vertices. By definition of total graph of a graph, we note that, $T\left(C_{n}\right)$ of $C_{n}$ is a 4- regular graph with $2 n$ vertices and each vertex will be of degree 4 . Thus $V\left(T\left(C_{n}\right) ; x\right)=2 n x^{4}$.

Corollary 3.17: The vertex polynomial root of $T\left(C_{n}\right)$ is 0 with multiplicity 4.

Proof: In view of Theorem 3.16, result is obvious.

Theorem 3.18: If $T\left(W_{n}\right)$ is the total graph of $W_{n}$, then $V\left(T\left(W_{n} ; x\right)=(2 n-2) x^{6}+(n-1) x^{n+2}+x^{2 n-2}\right.$, for $n \geq 5$

Proof: We know a wheel graph $W_{n}$ has $n$ vertices and $2 n-$ 2 edges. By definition of total graph, we note that $T\left(W_{n}\right)$ of $W_{n}$ will have $(3 n-2)$ vertices and we can partition this vertex set into three disjoint subsets $V_{1}, V_{2}$ and $V_{3}$ as follows:

$V_{1}$ : Vertex set containing $(2 n-2)$ vertices, each of degree 6 ,

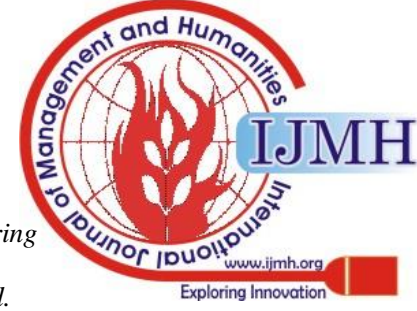


$V_{2}$ : Vertex set containing a single vertex of degree $(2 n-2)$ and

$V_{3}$ : Vertex set containing $(n-1)$ vertices, each of degree $(n+2)$.

Thus $V\left(T\left(W_{n} ; x\right)=(2 n-2) x^{6}+(n-1) x^{n+2}+x^{2 n-2}\right.$

\section{IV.CONCLUSION}

Vertex polynomial is one of the possible ways in which we can represent a graph algebraically. We can compare this polynomials with other polynomials associated with graph also.

\section{REFERENCES}

1. F.Haray, Graph Theory, Addison-Wesley, Reading- Mass (1969).

2. J.Devaraj and E.Sukumaran, On Vertex Polynomial, International J. of Math. Sci. \& Engg. Appls. (IJMSEA), Vol. 6 No. I (January, 2012), pp. 371-380.

3. A.M.Anto and P.Paul Hawkins, Vertex Polynomial of Graphs with New Results, Global Journal of Pure and Applied Mathematics, Volume 15, Number 4 (2019), pp. 469-475.

4. Mallikarjun Basanna Kattimani and Sridhara K.R., Transitive domination polynomial in graphs, Studies in Indian Place Names (UGC Care Journal), ISSN: 2394-3114, Vol-40-Issue-70-March 2020, pp. 440-447.

\section{AUTHORS' PROFILE}

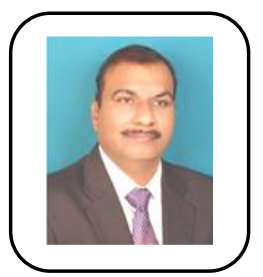

Dr.Mallikarjun Basanna Kattimani , $\mathrm{He}$ is working as Professor and Head, Department of Mathematics \& Dean of Examinations at The Oxford College of Engineering, Bangalore. His current area of research interest is Graph Theory. He has successfully guided one student for Ph.D. and presently guiding 6 students for Ph.D. He has introduced many domination parameters in Graph Theory and has published 12 research papers in national and international journals. In addition, he has 7 extended research articles to his credit. He has around 28 years of rich teaching experience.

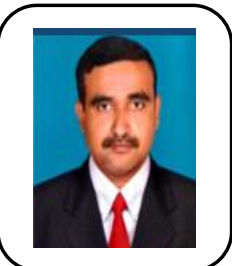

Sridhara K.R., He is working as Assistant Professor and Head, Department of Mathematics, A.P.S. College of Engineering, Bangalore. His research area of interest is Graph Theory. He has around 21 years of teaching experience. 